\title{
Differences in the Upper-Limb Coordination of Primary School Children
}

\author{
Miljan Hadzovic, Predrag Ilic, Aleksandra Aleksic Veljkovic \\ University of Nǐ̌, Niš, Serbia
}

\begin{abstract}
Background. Monitoring the abilities of typically developed children is very important.

Methods. This study aimed to determine the levels and differences in motor coordination of the upper extremities between first and third-grade primary school children using the subtest of the Bruininks-Oseretsky Test (BOT-2). The sample included 79 healthy subjects of both sexes, chronological age ranging from seven to nine years, body height $134.51 \pm 8.43 \mathrm{~cm}$ (Mean $\pm S D$ ), bodyweight $33.01 \pm 8.65 \mathrm{~kg}$ (Mean $\pm S D$ ). The Mann-Whitney $U$ test determined differences between groups, for the benefit of the group of third-grade subjects in all upper extremity coordination variables.

Results. The results showed that differences in age and biological growth and development in young children in some variables of upper-limb coordination influenced medium size (2ULC, 6ULC, 7ULC), but in most of the variables examined they had great influence (1ULC. 3ULC, 4ULC, 5ULC, TULC) according to Cohen's criterion.

Conclusion. It can be concluded that well-organized and expertly guided physical activities, as well as regular testing of children when it comes to developing coordination, can influence early detection of movement difficulties and eliminate problems in acquiring new coordination skills, and thus create an appropriate base for engaging in sports activities while growing up.
\end{abstract}

Keywords: motor development, coordination, physical education.

\section{INTRODUCTION}

$\mathrm{M}$ otor development, especially in the early stages, is one of the main indicators in assessing the proper development of children in the future. Motor problems may be associated with insufficient physical activity, the onset of obesity, poor concentration, and academic success, as well as other children's psychosociological competences (Venetsanou, Kambas, Aggeloussis, Fatouros, \& Taxildaris, 2007). During the period of growth and development in children, morphological and physiological changes occur (Herodek, Zivkovic, \& Aleksic-Veljkovic, 2019), as well as the creation of motor stereotype in children through the development of motor skills. The question is whether the personality is under the influence of motor learning and whether there is a basis for the creation of an adequate capacity to participate in a variety of physical activity in the period of growth (Williams et al., 2008).

Dealing with various motor tasks requires a certain level of development of general motor skills, which implies the equal development of gross motor skills - to initiate different movements by activating large muscle groups and fine motor skills responsible for coordinating the manipulation of small objects with the help of smaller muscles or smaller muscle groups (Goodway, Ozmun, \& Gallahue, 2019). Several studies have examined motor coordination in young children without the impaired motor development using different tests adapted for childhood (Cairney et al., 2005; Cairney, Hay, Veldhuizen, Missiuna, \& Faught, 2010; Dighe et al., 2017; Morales, González, Guerra, Virgili, \& Unnithan, 2011; da Silva Pacheco, Gabbard, Ries, 
\& Bobbio, 2016; Vandendriessche et al., 2011; Vandorpe et al., 2011), but also for children with a specific type of disability (Aki, Atasavun, Turan, \& Kayihan, 2007; Kaur, Srinivasan, \& Bhat, 2018; Pan et al., 2017; Rutkowska et al., 2016; Wuang \& Su, 2009). A test that has been validated (Balakrishnan $\&$ Rao, 2007) and is most commonly used to assess motor coordination in children is the BruininksOseretsky Test (BOT), and its use is recommended as a standardized measure of the level of motor skills of children by physical education professors and physical therapists (Deitz, Kartin, \& Kopp, 2007). A review of literature dealing with the problem of coordination as a motor ability in children revealed a lack of research solely dealing with the influence of biological growth and development on differences in coordination performance of students in young school age, that is, in children aged seven to nine years. Therefore, the question is whether there is a statistically significant difference in the levels of development of upper extremity coordination in children and how much structural and functional changes during adulthood and early education affect the motor coordination of children.

This experimental study aimed to determine the levels and differences in motor coordination of the upper extremities between first and third-grade primary school children using the BruininksOseretsky Test (BOT-2). Assuming that there is a statistically significant difference in upper extremity coordination between the two ages of children and that proper development during early schooling contributes to and promotes coordination, testing of children was performed using BOT-2 upper limb coordination subtests (Subtest 7, Upper-Limb Coordination).

\section{METHODS}

Respondents. In this cross-sectional study, a sample of respondents consisted of students of the first and third grades of the elementary school "Miroslav Antic" from Niš. The sample included 79 healthy subjects of both sexes, chronological age ranging from seven to nine years, body height $(\mathrm{BH})$ ranging from 117 to $154 \mathrm{~cm}$ (Mean $\pm S D: 134.51 \pm$ $8.43 \mathrm{~cm}$ ), minimum body mass (BW) of $21.3 \mathrm{~kg}$ to a maximum of $57 \mathrm{~kg}($ Mean $\pm S D: 33.01 \pm 8.65 \mathrm{~kg})$ and with a body mass index (BMI) ranging from 13.7 to $28.5 \mathrm{~kg} / \mathrm{m}^{2}$ (Mean $\pm S D: 18.02 \pm 3.20 \mathrm{~kg} / \mathrm{m}^{2}$ ). All subjects were clinically healthy and at the time of measurement had no obstruction of the locomotor apparatus or any other medical interference that would limit or interfere with the performance of the tests. The parents gave their consent for their children to be tested and all subjects voluntarily participated in the measurement and testing. The subjects were divided into two groups, the first group FG consisting of 35 first grade students (Mean \pm $S D: \mathrm{BH}=127.12 \pm 4.50 \mathrm{~cm}, \mathrm{BW}=26.91 \pm 3.96 \mathrm{~kg}$, $\mathrm{BMI}=16.59 \pm 1.76 \mathrm{k} / \mathrm{m}^{2}$ ) and the second group TG which consisted of 44 third grade students (Mean \pm $S D: \mathrm{BH}=140.39 \pm 5.76 \mathrm{~cm}, \mathrm{BW}=37.86 \pm 8.30 \mathrm{~kg}$, BMI $\left.=19.15 \pm 3.62 \mathrm{~kg} / \mathrm{m}^{2}\right)$. All subjects participated in physical activities covered by physical education classes with a frequency of three hours per week for 45 minutes.

Procedures. The testing of participants in this experimental study was conducted in the gymnasium of the elementary school "Miroslav Antic" in Niš in the morning to exclude daily variations of measurements. The room air temperature during testing ranged from 22 to $26^{\circ} \mathrm{C}$. Following the set research goal, the following morphological characteristics were measured: body height $(\mathrm{BH})$ using an anthropometer according to Martin with an accuracy of $0.1 \mathrm{~cm}$, and body weight (BW) using the electronic scale Body Composition Monitor BF511 (OMRON) for measurement accuracy in $0.1 \mathrm{~kg}$. Body mass index (BMI) was calculated based on the formula by which body weight in kilograms is divided by squared body height in meters $\left(\mathrm{BMI}=\mathrm{BW}(\mathrm{kg}) / \mathrm{BH}\left(\mathrm{m}^{2}\right)\right)$.

To determine the level of coordination of the subjects, an abbreviated BOT-2 test was applied (R. Bruininks \& B. Bruininks, 2005), or subtest to assess motor coordination of the upper extremities (Subtest 7, Upper-Limb Coordination). Using the tasks of the applied test, the measurement of the subjects collected the data of the following variables: dropping and catching the ball with both hands (1ULC), catching the thrown ball at a proper distance by the meter with both hands (2ULC), dropping and catching the ball with one hand (3ULC), catching the thrown ball at a proper distance by the meter with one hand (4ULC), dribbling with one hand (5ULC), dribbling with both hands (6ULC), the precision of aiming and shooting the ball in the target (7ULC) and overall coordination of the upper extremities of the subjects (TULC).

Description and operating conditions of the experiment. In this study, a cross-sectional research model was applied. Testing of a sample of 
children was conducted on November 29, 2019, from 10:45 a.m. to 12:15 p.m., and December 3, 2019, from 1:00 p.m. to 1:45 p.m., during regular physical education classes. Respondents were verbally explained and practically demonstrated how to perform the test before starting the test. Before the test began, the subjects, under the guidance of the professor, performed warm-up exercises for 10 minutes. All subjects were measured under identical conditions and reported to be measured and tested in the same order under the direction of the meters. During the measurements, the respondents were without shoes and during testing - in sports clothes ready for physical education. Measurement and testing were performed by experienced assistants, who were thoroughly familiarized with the protocol before measuring and testing. The survey was conducted following the principles of the Declaration of Helsinki.

Statistical analysis. All obtained data are represented by parameters of descriptive statistics (mean-mean, standard deviation - SD, minimum result - min and maximum result - max). The Kolmogorov-Smirnov test was used to evaluate the normality of the distribution of results. MannWhitney $U$ test was used to determine differences between groups. For the statistical significance of differences in scores between groups, a significance level of $p<.05$ was used. Data were processed in the SPSS statistical package (IBM Corp. Released 2010. IBM SPSS Statistics for Windows, Version 19.0. Armonk, NY: IBM Corp.).

\section{RESULTS AND DISCUSSION}

The basic statistics (number of students and gender representation) are shown in Table 1.

After completing the testing on the collected data using the Kolmogorov-Smirnov test, an analysis of the normality of the distribution of results was performed. The assumption of normality of the distribution of results was rejected because in all tested variables statistical significance of differences at $p<.05$ was established (Table 2)

Further analysis of the results, using the MannWhitney $U$ test to determine differences between groups, determined statistical significance at $p<.05$ for the benefit of the group of third-grade subjects in all upper extremity coordination variables tested (Table 3).

The magnitude of the influence $(r)$ of the biological growth and development of the subjects on the measurements covered by the variables was calculated using the formula $r=Z / \sqrt{ } N$ (where $N$ is the total number of subjects) according to Cohen's criterion $(.1=$ small influence; .3 = mean influence; $.5=$ high impact) and is presented in a table (Table 4).

The results presented in Table No. 6 showed that differences in age and biological growth and development in young children in some variables influenced medium size (2ULC, 6ULC, 7ULC), but in most of the variables examined they had great influence (1ULC. 3ULC, 4ULC, 5ULC, TULC).
Table 1. Number and percentage of sample by gender

\begin{tabular}{|l|c|c|c|}
\hline Number of students & Male & Female & Total number of students \\
\hline First grade & $20(57.14 \%)$ & $15(42.86 \%)$ & 35 \\
\hline Third grade & $21(47.73 \%)$ & $23(52.27 \%)$ & 44 \\
\hline
\end{tabular}

Table 2. Normality of distribution

\begin{tabular}{|c|c|c|c|}
\hline \multirow{2}{*}{ Variables } & \multicolumn{3}{|c|}{ Kolmogorov-Smirnova $^{\mathbf{a}}$} \\
\cline { 2 - 4 } & Statistic & $\boldsymbol{d f}$ & Sig. \\
\hline 1ULC & .45 & 79 & .00 \\
\hline 2ULC & .36 & 79 & .00 \\
\hline 3ULC & .34 & 79 & .00 \\
\hline 4ULC & .17 & 79 & .00 \\
\hline 5ULC & .24 & 79 & .00 \\
\hline 6ULC & .36 & 79 & .00 \\
\hline 7ULC & .15 & 79 & .00 \\
\hline TULC & .11 & 79 & .03 \\
\hline
\end{tabular}


Table 3. Mann-Whitney $\boldsymbol{U}$ Test

\begin{tabular}{|l|c|c|c|c|c|c|c|c|}
\hline \multicolumn{1}{|c|}{ Variable } & 1ULC & 2ULC & 3ULC & 4ULC & 5ULC & 6ULC & 7ULC & TULC \\
\hline Mann-Whitney $U$ & 374.00 & 415.50 & 344.50 & 259.00 & 266.00 & 377.00 & 458.500 & 148.50 \\
\hline Wilcoxon $W$ & 1004.00 & 1045.50 & 974.50 & 889.00 & 896.00 & 1007.00 & 1088.50 & 778.50 \\
\hline$Z$ & -5.33 & -4.02 & -4.79 & -5.13 & -5.15 & -3.95 & -3.16 & -6.14 \\
\hline Asymp. Sig. (2-tailed) & .00 & .00 & .00 & .00 & .00 & .00 & .02 & .00 \\
\hline
\end{tabular}

Table 4. The magnitude of the impact of biological growth and development in young children on the examined variables expressed by the Cohen criterion

\begin{tabular}{|c|c|c|c|}
\hline Variable & $\boldsymbol{r}=\boldsymbol{Z} / \sqrt{\boldsymbol{N}}$ & $\boldsymbol{r}$ & Impact \\
\hline 1ULC & $r=-5.33 / \sqrt{79}=-.60$ & $>.5$ & High impact \\
\hline 2ULC & $r=-4.02 / \sqrt{79}=-.45$ & $.3-.5$ & Medium impact \\
\hline 3ULC & $r=-4.79 / \sqrt{79}=-.54$ & $>.5$ & High impact \\
\hline 4ULC & $r=-5.13 / \sqrt{79}=-.58$ & $>.5$ & High impact \\
\hline $5 \mathrm{ULC}$ & $r=-5.15 / \sqrt{79}=-.58$ & $>.5$ & High impact \\
\hline 6ULC & $r=-3.95 / \sqrt{79}=-.44$ & $.3-.5$ & Medium impact \\
\hline 7ULC & $r=-3.16 / \sqrt{79}=-.36$ & $.3-.5$ & Medium impact \\
\hline TULC & $r=-6.14 / \sqrt{79}=-.69$ & $>.5$ & High impact \\
\hline
\end{tabular}

The development of motor skills and the evolution of skills that are structured around motor coordination, especially during primary education, is extremely important as it provides an excellent basis for the proper development of each individual (Barela, 2013). According to some authors, the development of motor coordination and coordination skills occur at different times and improve in different ways as they grow up, and that most progress is generally made in the period between the age of four and puberty (Weineck, 2005). The purpose of this experimental study was to determine the developmental level of coordination in young school-age children and to determine differences in upper-extremity coordination between first (FG) and third (TG) grade students using the BOT-2 subtest to assess motor coordination of upper extremities, the consequences of biological growth and the development during schooling.

The results of the tests used in this study, in mixed groups of children of different ages $(\mathrm{FG}=$ $7 \mathrm{y} ; \mathrm{TG}=9 \mathrm{y}$ ), showed unequivocally that there were differences in the level of upper extremity coordination achieved in favour of older children (TG), which agrees with the authors' claims in previous studies that results achieved in motor coordination increase with morphological changes during growth and development of children
(Vandendriessche et al., 2011; Dighe et al., 2017). These claims about the results obtained were confirmed using the Mann-Whitney U Test, which showed statistical significance in all variables at the $p<.05$ level in favour of the TG group of subjects (1ULC: $p=.00$; 2ULC: $p=.00$; 3ULC: $p=.00$; 4ULC: $p=.00 ;$ 5ULC: $p=.00$; 6ULC: $p=.00$; 7ULC: $p=.02$; TULC: $p=.00$ ).

This research confirmed the claims of Dighe et al. (2017) that morphological characteristics, as a reflection of biological growth and development during adulthood, are indicated by differences in mean $\mathrm{BH}, \mathrm{BW}$, and $\mathrm{BMI}$ values in favour of thirdgrade students $(\mathrm{TG}: \mathrm{BH}=140.39 \pm 5.76 \mathrm{~cm}, \mathrm{BW}=$ $37.86 \pm 8.30 \mathrm{~kg}, \mathrm{BMI}=19.15 \pm 3.62 \mathrm{~kg} / \mathrm{m}^{2}$; according to $\mathrm{FG}: \mathrm{BH}=127.12 \pm 4.50 \mathrm{~cm}, \mathrm{BW}=26.91 \pm$ $3.96 \mathrm{~kg}, \mathrm{BMI}=16.59 \pm 1.76 \mathrm{~kg} / \mathrm{m}^{2}$ ), indicating, above all, limb length, general musculature, and neuromuscular coordination, structural and functional differences due to advancing age in children may be the reason for better coordination performance.

The magnitude of the impact of biological growth and development on coordination in children of different ages in the early elementary education period was estimated according to the Cohen criterion. The results after testing showed that the highest influence $(r>.5)$ was achieved in 
the overall coordination of the upper extremities (TULC: $r=-.69$ ). Also, differences in age of children are greatly influenced by most variables such as dropping and catching the ball once (3ULC: $r=-.54$ ) and with both hands (1ULC: $r=-.60$ ), catching the thrown ball at a proper distance by the meter one-handed (4ULC: $r=-.58$ ) and one-handed dribbling (5ULC: $r=-.58$ ) and that means influence $(r=.3-.5)$ have on variables such as catching a thrown ball from a proper distance by the meter with both hands (2ULC: $r=-.45$ ), dribbling balls with both hands (2ULC: $r=-.44$ ) and precision targeting and shooting the ball at target (7ULC: $r=-.36$ ).

Although this study is limited when it comes to data on the extracurricular sports and recreational activities of the respondents, the results obtained when it comes to the coordination of the upper extremities can be explained, in addition to differences in growth and development, by the longer period of attendance of physical education of older children, which is according to the author of one of the studies that regular, adequately planned and professionally guided physical activity during early education can contribute to the proper development of basic motor skills and coordination performance NSI, which will cause thus demanding their inclusion in sports activities during adulthood (Barela, 2013).

The results of this cross-sectional study, conducted on children of different ages of seven and nine years, confirmed the existence of differences in the level achieved and the development of motor coordination of the upper extremities. A detailed analysis of the results revealed that there were statistically significant differences reflected in the overall coordination of the upper extremities, as well as in all testing included variables of this motor ability, and that differences in age, morphological characteristics and biological growth and development have a great impact when it comes to the development of motor coordination in young children. It can also be concluded that wellorganized and expertly guided physical activities, as well as regular testing of children when it comes to developing coordination, can influence early detection of movement difficulties and eliminate problems in acquiring new coordination skills, and thus create an appropriate base for engaging in sports activities while growing up.

\section{CONCLUSION}

Based on the results and limitations of this cross-sectional study, as well as the conclusions of previous studies, recommendations for further research are reflected in the increase in the sample of respondents by including children from different social groups. Also, longitudinal research needs to be done to evaluate specific training programs to develop coordination while increasing variables by including tests for fine motor coordination.

\section{REFERENCES}

Aki, E., Atasavun, S., Turan, A., \& Kayihan, H. (2007). Training motor skills of children with low vision. Perceptual and Motor Skills, 104(Suppl. 3), 1328-1336. https://doi.org/10.2466/pms.104.4.1328-1336

Balakrishnan, T., \& Rao, C. S. (2007). Interrater reliability of bilateral coordination of Bruininks Oseretsky Test of Motor Proficiency (BOTMP) \& Performance of Indian Children compared with USA norms. The Indian Journal of Occupational Therapy, 38(3), 55-60. Retrieved from http://eprints.manipal.edu/id/eprint/805

Barela, J. A. (2013). Fundamental motor skill proficiency is necessary for children's motor activity inclusion. Motriz: Revista de Educação Física, 19(3), 548-551. doi: 10.1590/S1980-65742013000300003

Bruininks, R., \& Bruininks, B. (2005). BruininksOseretsky test of motor proficiency (2nd ed.). Minneapolis, MN: NCS Pearson.

Cairney, J., Hay, J. A., Faught, B. E., Wade, T. J., Corna, L., \& Flouris, A. (2005). Developmental coordina- tion disorder generalized self-efficacy toward physical activity, and participation in organized and free play activities. The Journal of Pediatrics, 147(4), 515-520. https://doi.org/10.1016/j.jpeds.2005.05.013

Cairney, J., Hay, J. A., Veldhuizen, S., Missiuna, C., \& Faught, B. E. (2010). Developmental coordination disorder, sex, and activity deficit over time: A longitudinal analysis of participation trajectories in children with and without coordination difficulties. Developmental Medicine \& Child Neurology, 52(3), e67-e72. https:// dx.doi.org/10.1177\%2F2333794X19845920

da Silva Pacheco, S. C., Gabbard, C., Ries, L. G. K., \& Bobbio, T. G. (2016). Interlimb coordination and academic performance in elementary school children. Pediatrics International, 58(10), 967-973. https://doi. org/10.1111/ped.12972

Deitz, J. C., Kartin, D., \& Kopp, K. (2007). Review of the Bruininks-Oseretsky test of motor proficiency (BOT-2). Physical \& Occupational 
Therapy in Pediatrics, 27(4), 87-102. https://dx.doi. org/10.1186\%2F1471-2431-13-135

Dighe, A. D., Dhote, D., Palekar, D., Pande, A., Yengde, P., \& Singh, G. (2017). Assessment of upper limb coordination using Bruininks-Oseretsky test of motor proficiency, (bot-2), in 5-15 years of schoolgoing, children. International Journal of Healthcare and Biomedical Research, 5(3), 62-69.

Goodway, J. D., Ozmun, J. C., \& Gallahue, D. L. (2019). Understanding motor development: Infants, children, adolescents, adults. Burlington: Jones \& Bartlett Publishers.

Herodek, K., Živković, M., Aleksić-Veljković, A. (2019). Motor development, Niš, RS: Faculty of Sport and Physical Education, University of Nis.

Horvat, V., Babić, V., \& Miholić, S. J. (2013). Gender differences in some motor abilities of preschool children. Croatian Journal of Education, 15(4), 959980.

Kaur, M., Srinivasan, S. M., \& Bhat, A. N. (2018). Comparing motor performance, praxis, coordination, and interpersonal synchrony between children with and without Autism Spectrum Disorder (ASD). Research in Developmental Disabilities, 72, 79-95. https://doi. org/10.1016/j.ridd.2017.10.025

Morales, J., González, L. M., Guerra, M., Virgili, C., \& Unnithan, V. (2011). Physical activity, perceptualmotor performance, and academic learning in 9-to-16years-old school children. International Journal of Sport Psychology, 42(4), 401-415.

Pan, C. Y., Chu, C. H., Tsai, C. L., Sung, M. C., Huang, C. Y., \& Ma, W. Y. (2017). The impacts of physical activity intervention on physical and cognitive outcomes in children with an autism spectrum disorder. Autism, 21(2), 190-202. https://doi. org/10.1177/1362361316633562
Rutkowska,I.,Lieberman,L.J., Bednarczuk, G., Molik,B., Kazimierska-Kowalewska, K., Marszałek, J., \& GómezRuano, M. Á. (2016). Bilateral coordination of children who are blind. Perceptual and Motor Skills, 122(2), 595609. https://doi.org/10.1177\%2F0031512516636527

Vandendriessche, J. B., Vandorpe, B., Coelho-eSilva, M. J., Vaeyens, R., Lenoir, M., Lefevre, J., \& Philippaerts, R. M. (2011). Multivariate association among morphology, fitness, and motor coordination characteristics in boys age 7 to 11. Pediatric Exercise Science, 23(4), 504-520.

Vandorpe, B., Vandendriessche, J., Lefèvre, J., Pion, J., Vaeyens, R., Matthys, S., Philippaerts, R., \& Lenoir, M. (2011). The Körperkoordinationstest für kinder: Reference values and suitability for 6-12-year-old children in Flanders. Scandinavian Journal of Medicine \& Science in Sports, 21(3), 378-388. https://doi. org/10.1111/j.1600-0838.2009.01067.x

Venetsanou, F., Kambas, A., Aggeloussis, N., Serbezis, V., \& Taxildaris, K. (2007). Use of the Bruininks-Oseretsky test of motor proficiency for identifying children with motor impairment. Developmental Medicine \& Child Neurology, 49(11), 846-848. https://doi.org/10.1111/ j.1469-8749.2007.00846.x

Weineck, J. (2005). Entrenamiento in total. Badalona (Barcelona): Paidotribo.

Williams, H. G., Pfeiffer, K. A., O'neill, J. R., Dowda, M., McIver, K. L., Brown, W. H., \& Pate, R. R. (2008). Motor skill performance and physical activity in preschool children. Obesity, 16(6), 1421-1426. https:// doi.org/10.1038/oby.2008.214

Wuang, Y. P., \& Su, C. Y. (2009). Reliability and responsiveness of the Bruininks-Oseretsky Test of Motor Proficiency-in children with intellectual disability. Research in Developmental Disabilities, 30(5), 847-855. https://doi.org/10.1016/j.ridd.2008.12.002 